\title{
Biologically-Inspired Peptide Reagents for Enhancing IMS-MS Analysis of Carbohydrates
}

\author{
Brian C. Bohrer, David E. Clemmer
}

Department of Chemistry, Indiana University, Bloomington, IN 47405, USA

\begin{abstract}
The binding properties of a peptidoglycan recognition protein are translated via combinatorial chemistry into short peptides. Non-adjacent histidine, tyrosine, and arginine residues in the protein's binding cleft that associate specifically with the glycan moiety of a peptidoglycan substrate are incorporated into linear sequences creating a library of 27 candidate tripeptide reagents (three possible residues permutated across three positions). Upon electrospraying the peptide library and carbohydrate mixtures, some noncovalent complexes are observed. The binding efficiencies of the peptides vary according to their amino acid composition as well as the disaccharide linkage and carbohydrate ring-type. In addition to providing a charge-carrier for the carbohydrate, peptide reagents can also be used to differentiate carbohydrate isomers by ion mobility spectrometry. The utility of these peptide reagents as a means of enhancing ion mobility analysis of carbohydrates is illustrated by examining four glucose-containing disaccharide isomers, including a pair that is not resolved by ion mobility alone. The specificity and stoichiometry of the peptide-carbohydrate complexes are also investigated. Trihistidine demonstrates both suitable binding efficiency and successful resolution of disaccharides isomers, suggesting it may be a useful reagent in IMS analyses of carbohydrates.
\end{abstract}

Key words: Ion mobility spectrometry, Carbohydrates, Shift reagents, Noncovalent complexes

\section{Introduction}

G lycosylation is a ubiquitous post-translational modification that is thought to influence the structures of roughly half of all proteins [1]. Analysis of the positions of and structures of glycans on protein surfaces is tremendously challenging because of a number of reasons, including: the branched nature of glycans, leading to a large number of possible isomeric forms; the heterogeneity of glycosylation (both positional and structural variations) among many copies of the same protein; and, the limited quantities of samples where glycan analysis is desirable. Mass spectrometry (MS)-based techniques and affinity-based enrichment approaches [2-4] are among the most promising solutions to these problems. For example, Reinhold and coworkers have utilized multiple stages of tandem mass spectrometry (up to

Correspondence to: Brian C. Bohrer; e-mail: clemmer@indiana.edu
$\mathrm{MS}^{6}$ ) to determine the structures of isomeric glycans from ovalbumin $\operatorname{IgG}$ [5]. The combination of collision induced dissociation (CID) with electron transfer dissociation (ETD) is making it possible to determine peptide sequences as well as glycan positions and structures [3]. Recently, ion mobility spectrometry (IMS) [6] has emerged as a means of separating isomers prior to MS analysis [7-16]. Because IMS separates ions based on their shapes, it also offers information that is complementary to MS analysis. By comparing experimentally measured mobilities with those calculated for trial structures generated by theory it is possible to gain insight into the shapes of different glycan isomer forms.

Some isomers, however, are too similar in size to be distinguishable even at the resolving power of current stateof-the-art IMS instruments. Several strategies have been proposed to resolve such species. One approach is to change the composition of the buffer gas used inside the drift cell of 
an ion mobility spectrometer. Changing the buffer gas or doping in gaseous chemical modifiers has been shown to significantly affect the resolution of several analytes [17-19] and this strategy has been used successfully for carbohydrates as well [12]. Another route has been to vary the ionization state of the analyte species. Because saccharidebased analytes do not typically protonate favorably, electrospray ionization (ESI) [20] typically involves salt-containing solutions to form metal cation adducts. Sodium salts are most typically used, although other alkali metals [16] and some transition metals [12] have been investigated.

Although they have yet to be used in IMS studies, Desaire and coworkers have shown that ion-pairing reagents can be used to bind and ionize certain functionalized (e.g., phosphorylated or sulfated) saccharide species [21, 22]. Due to the acidity of these functional groups, such ion-pairing reagents are typically oligomers of basic amino acid residues, such as trilysine [22]. These reagents make it possible to differentiate between phosphorylated and sulfated species based on characteristic fragment ions produced upon collision-induced dissociation [21]. Peptide-based reagents are also appealing because, unlike metal cations, they have inherent structure that may be flexible. Therefore, it seems likely that appropriate peptide reagents may distinguish between isomeric substrate molecules based on their binding affinities or by the geometries of the complexes they form. A recent report suggests that peptide-carbohydrate interactions are capable of distinguishing between different anomeric forms of a saccharide molecule [23]. We also note the related work by Cotter and Von Seggern utilizing biologically-relevant interactions between peptides and carbohydrates in mass spectrometry experiments [24, 25].

Here, we describe an approach that utilizes known binding interactions from biological systems to design peptide-based reagents for improved resolution and identification of carbohydrates. The candidate peptide reagents are modeled after the binding cleft of a peptidoglycan recognition protein (PGRP) [26]. This protein has been shown to bind to a muramyl tripeptide that serves to model the peptidoglycan layer of bacterial cell walls [27]. Both the carbohydrate and peptide portions of the substrate are required for binding [26], suggesting that a region of the binding cleft interacts specifically with the glycan moiety. In a co-crystallized PGRP-muramyl tripeptide complex, three amino acid residues were identified to be in close proximity to (and presumably associating with) the muramyl group [26]. These residues (histidine, tyrosine, and arginine) were incorporated in the design of a tripeptide library that contains all 27 possible combinations of the three amino acids. The resulting library is then doped into electrospray solutions of several saccharide-based analytes including $\mathrm{N}$-acetylmuramic acid, maltohexaose, and a set of four disaccharide isomers. These peptide reagents are investigated for saccharide-binding efficiency and stoichiometry, and isomeric tripeptide-disaccharide complexes are exam- ined to assess the suitability of these peptides as IMS shift reagents $[28,29]$.

\section{Experimental}

\section{Peptide Library Synthesis}

Peptides were synthesized via standard fluorenylmethyloxycarbonyl (Fmoc) chemistry and solid-phase synthesis procedures [30, 31]. All reagents were purchased from Midwest Bio-Tech, unless otherwise noted. The peptide chain was synthesized starting from a mixture of Wang resin beads, preloaded with Fmoc-protected His(Trt), Tyr(tBu), and $\operatorname{Arg}(\mathrm{Pbf})$ residues, which were deprotected with $20 \%$ piperidine in $N, N$-dimethylformamide (DMF) in two 10-min steps. Subsequent residues, Fmoc-protected His(Trt), Tyr $(\mathrm{tBu})$, and $\operatorname{Arg}(\mathrm{Pmc})$ (Nova Biochem), were coupled using 5-fold excess amino acid, HBTU, and DIPEA dissolved in DMF, and were allowed to react for $30 \mathrm{~min}$ with occasional agitation. After coupling, the resin beads were dried, divided equally by mass, and redistributed among the three reaction vessels. After the third residue was coupled and its $\mathrm{N}$ terminus deprotected, the peptides were cleaved using a cocktail of 95/2.5/2.5 TFA:water:phenol for $2 \mathrm{~h}$. The peptides were precipitated with diethyl ether, filtered, and redissolved in 5\% aqueous acetic acid. This solution was then frozen, lyophilized, and used directly without further purification.

\section{Instrumentation}

The studies presented below were conducted on a homebuilt IMS-MS instrument. This instrument is described in detail elsewhere [32]. Briefly, a continuous beam of electrosprayed [20] ions are trapped and accumulated in a source ion funnel [33] by rf potentials $(\sim 150 \mathrm{~V}$, peak-to-peak amplitude at $\sim 300 \mathrm{kHz}$ ). Periodically, ion packets are released in short (150 $\mu$ s-wide) pulses into a $\sim 3 \mathrm{~m}$ long stacked ring-electrode drift tube. The drift tube is filled with $\sim 3$ Torr He buffer gas at room temperature and a linear electric field of $10 \mathrm{~V} \cdot \mathrm{cm}^{-1}$ is applied down its axis. As the packet of ions traverses the drift tube, different ions separate according to the differences in their low-field mobilities through the gas [34-36] typical total drift times for the ions produced here are on the order of tens of milliseconds.

Three additional ion funnels are located inside the drift tube and at the drift tube exit. These funnels are used to radially refocus the diffusing ion cloud. After the mobility separation, a series of ion optics (including deflectors, an Einzel lens assembly, and a short quadrupole) focus the ions through differential pumping stages and into the source of a time-of-flight (TOF) mass analyzer for rapid (maximum flight time of $60 \mu \mathrm{s}) \mathrm{m} / \mathrm{z}$ measurement. Roughly $10^{2}-10^{3}$ mass spectra are collected per IMS experiment, allowing drift time distributions to be constructed from individual TOF experiments in a nested [drift time(flight time)] fashion 
[37]. Flight times are converted into $\mathrm{m} / \mathrm{z}$ by a simple secondorder polynomial calibration.

\section{Electrospray Conditions}

Stock solutions of leucrose (5-O- $\alpha$-D-glucopyranosyl-Dfructose), melibiose (6-O- $\alpha$-D-galactopyranosyl-D-glucose), palatinose (6-O- $\alpha$-D-glucopyranosyl-D-fructose), and trehalose ( $\alpha$-D-Glucopyranosyl- $\alpha$-D-glucopyranoside) were obtained at $98 \%$ purity or greater from Fluka and prepared at $150 \mathrm{mM}$ in HPLC grade water. Electrospray solutions were then prepared by dilution to $3 \times 10^{-4} \mathrm{M}$ in $50 / 50$ water: acetonitrile (vol/vol) with $2 \mathrm{mM}$ sodium acetate. For analysis with peptide reagents, sodium acetate was substituted with $1 \%$ acetic acid and peptides at $8 \times 10^{-5} \mathrm{M}$ (unique peptide concentration). A syringe pump (KD Scientific) provided flow of the solution through a pulled capillary tip $(100 \mu \mathrm{m}$ i.d. $\times 360 \mu \mathrm{m}$ o.d. $)$ biased $\sim 2.2 \mathrm{kV}$ above the drift voltage at a flow rate of $0.25 \mu \mathrm{L} \cdot \mathrm{min}^{-1}$.

\section{Results and Discussion}

\section{IMS-MS Analysis of Disaccharides}

Figure 1 shows ESI mass spectra for the individual disaccharides ionized by sodium adducts. Intense peaks corresponding to sodiated monomer, dimer, and trimer are observed for each disaccharide isomer $(\mathrm{m} / \mathrm{z} 365,707$, and 1049, respectively). Data corresponding to sodiated monomers can be extracted along the mobility dimension to produce drift time distributions for these ions (also shown in Figure 1). Leucrose and trehalose ions are adequately resolved from one another and from the peaks representing palatinose and melibiose. The latter pair, however, would not be resolved when analyzed as a mixture. It is interesting to point out that these two species are both $\alpha(1 \rightarrow 6)$ linked disaccharides, whereas leucrose and trehalose are $\alpha(1 \rightarrow 5)$ and $\alpha, \alpha(1 \rightarrow 1)$ linked, respectively. The monomeric subunits of melibiose are both aldohexoses (galactose and glucose), whereas palatinose contains both an aldohexose and ketohexose (glucose and fructose, respectively). This difference is not sufficient to distinguish them based on their mobilities at the current instrumental resolving power (typically $\mathrm{R}=50-120$ ).

\section{Screening Peptide Reagents Against Disaccharides}

In order to shift the mobilities of these ions, we have attempted to form peptide-disaccharide complexes that will have different mobilities for isomeric species. We begin by considering which peptides in the library bind to disaccharides. The library of potential peptide reagents was first analyzed with a mixture of all four disaccharides. A twodimensional plot of the IMS-MS data for this system is shown in Figure 2. The most intense features in the dataset correspond to singly- and doubly-protonated tripeptide ions, with many peaks associated with tripeptide-disaccharide complexes observed at lower abundance. The preferred binding stoichiometry of these complexes under the present experimental conditions is 1:1 tripeptide:disaccharide, and the overall complexes are doubly charged. Significant intensity is also detected for $[\mathrm{nM}+\text { tripeptide }+2 \mathrm{H}]^{2+}$ ions (where $\mathrm{M}$ corresponds to one of the four disaccharides and $n=1-4)$. There is also evidence for a series of $[\mathrm{nM}+\text { tripeptide }+3 \mathrm{H}]^{3+}$ ions, where $n=3-7$. The presence of these disaccharide clusters in these spectra is consistent with the relatively high disaccharide concentration $(1.2 \mathrm{mM}$ total saccharide concentration) and the known tendency of some of these molecules to self-associate in aqueous environments [38]. It is interesting to note that these clusters only incorporate a single tripeptide. Given the high ratio of disaccharides to tripeptides in these clusters, it is unlikely that the peptide reagent is interacting specifically with more than one disaccharide molecule. That is, a 1:1 tripeptide-disaccharide complex is formed with some specificity, and this complex goes on to seed a cluster by associating with additional disaccharide molecules through saccharide-saccharide interactions.

A semiquantitative understanding of the binding efficiency of the tripeptide reagents for the disaccharide molecules is obtained by analyzing each disaccharide individually in the tripeptide library matrix. The binding efficiency is assessed by dividing the total ion intensity of all tripeptide-disaccharide complex ions by the intensities of all peaks corresponding to tripeptide ions. This ratio is then divided by the molar excess of disaccharide relative to tripeptide concentration. The resulting data allows comparison of binding efficiency as a function of peptide composition. The plot of these results for each disaccharide is shown in Figure 3. The binding efficiencies vary according to the particular disaccharide and constituent residues of the peptide. For each disaccharide, however, optimal binding is consistently associated with histidine-rich sequences ( $\mathrm{HHH}$ and one or more of $\mathrm{HHY}, \mathrm{HYH}$, and $\mathrm{YHH}$ ) and to a peptide sequence comprising two tyrosine residues and one arginine residue. Binding efficiencies for these sequences are all in excess of $8 \%$; some complexes exceed $15 \%$. Peptides containing one of each possible amino acid residues demonstrate moderate binding. Given that the library design was based on a receptor for a functionalized aldohexose, this is not entirely surprising. The disaccharide that this library has the highest affinity for is melibiose, although the trihistidine-leucrose complex is also favored.

A dashed line of reference at the 10\% level in Figure 3 represents the upper limit of the binding efficiency associated with nonspecific protein-saccharide interactions, as estimated by Klassen and coworkers [39]. This comparison indicates that several of the tripeptides bind significantly more efficiently than would be expected from a solely nonspecific interaction. In particular, melibiose forms the most abundant complexes with these peptides, with only the sequence YYY failing to meet or surpass the $10 \%$ threshold. One possible factor in the favorable binding of melibiose may be the 

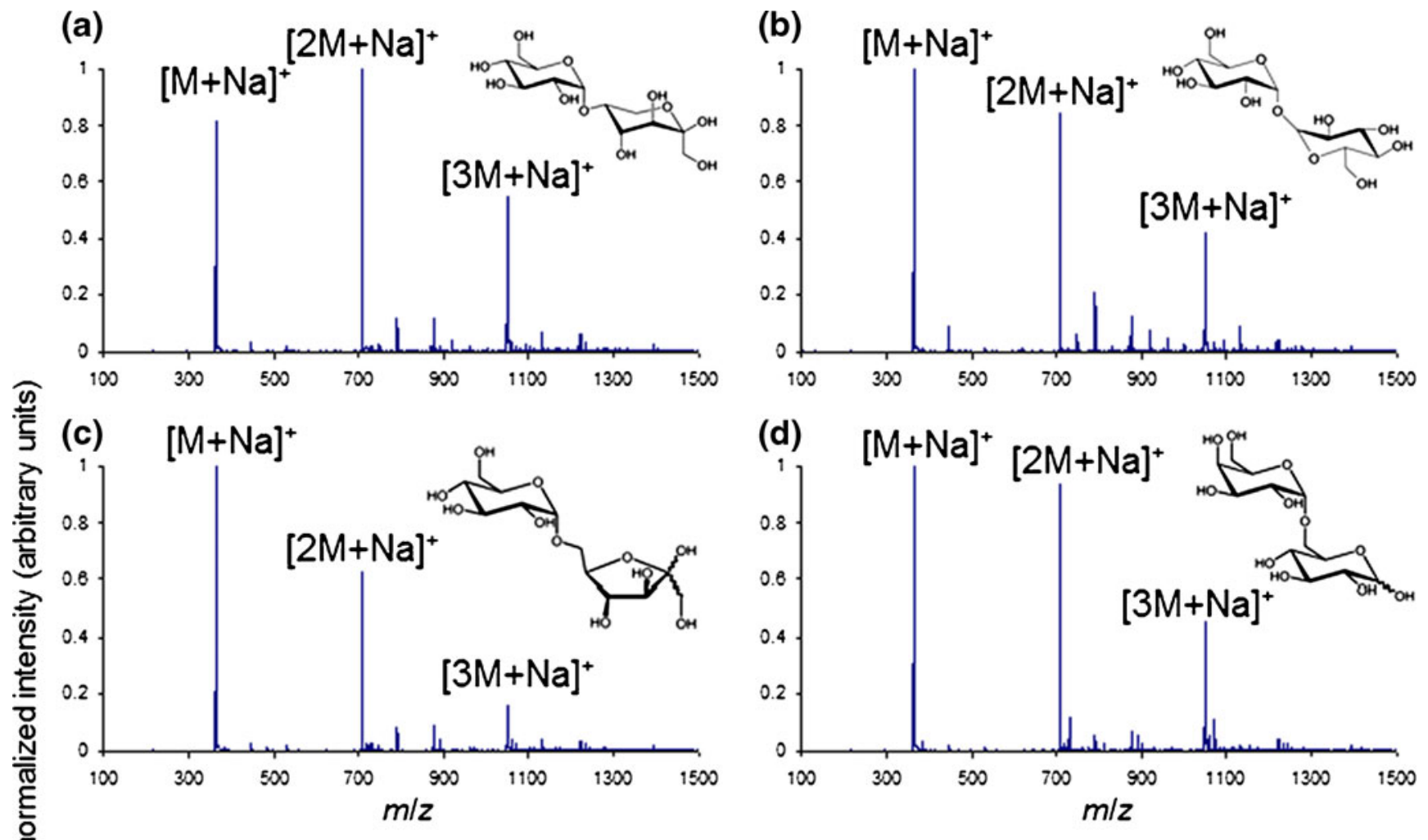

(e)

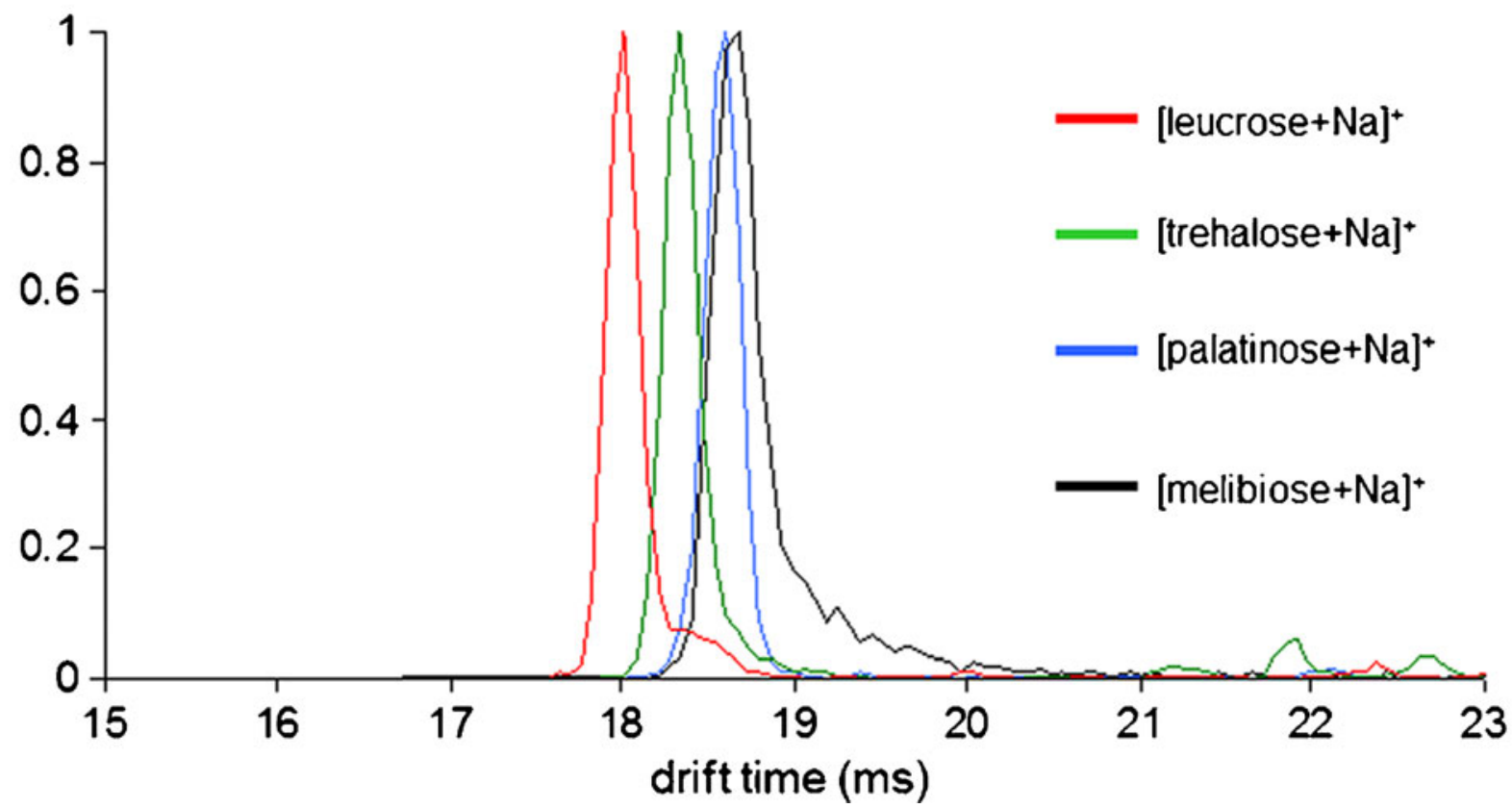

Figure 1. Sodium-cationized ESI mass spectra for individual disaccharides leucrose, trehalose, melibiose, and palatinose (a)(d), respectively. Drift time distributions for individual disaccharide monomers (e)

$\alpha(1 \rightarrow 6)$ linkage joining its aldohexose subunits. As demonstrated by the mobility of its sodium-cationized form, this linkage causes the disaccharide to adopt a relatively elongated form. Presumably, this conformation presents either of its subunits as available for recognition with minimal steric hindrance. Palatinose, the disaccharide that binds least efficiently (some complexes fail to exceed
$5 \%)$, is also a $\alpha(1 \rightarrow 6)$ linked disaccharide. However, it contains both an aldohexose and a ketohexose subunit. Furthermore, the fructose subunit of palatinose is found exclusively in its furanose form [40]. Therefore, its lower binding efficiency (approximately half the efficiency observed for melibiose and most peptide sequences) suggests that the tripeptides bind favorably only to 


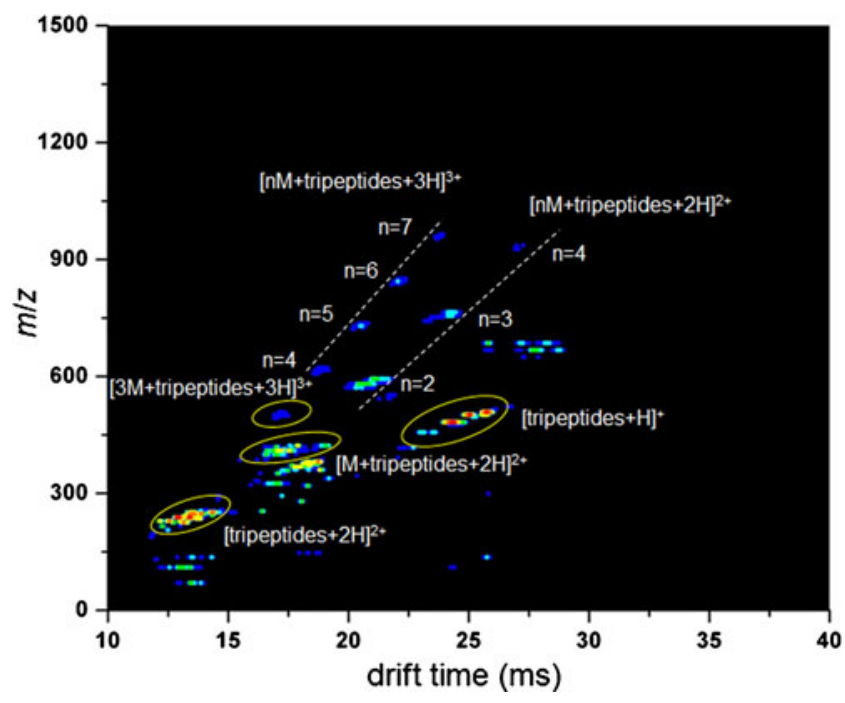

Figure 2. Two-dimensional IMS-MS plot of a 1:4 (unique peptide:unique disaccharide) molar ratio mixture. The complexes of lower stoichiometry are highlighted in yellow ovals, with dotted arrows showing the pattern indicative of additional disaccharide molecules (M) adding to a doubly and triply charged ion series

aldohexose moieties. Leucrose demonstrates the second-highest binding efficiency ( $10 \%$ average) and also contains a fructose unit on its reducing end. In this case, however, the fructose is found only in the pyranose form [40]. As such, the subunit more closely resembles an aldohexose moiety, and thus would alleviate the binding inefficiency that appears to be associated with furanose structures. Although the peptide-carbohydrate interactions are almost certainly dominated by hydrogen bonding, there remains a subtlety about the origin of favored peptide-carbohydrate pairs. Through the combinatorial methods employed here, however, we are able to explore the possible sequences and determine these favored pairs empirically.

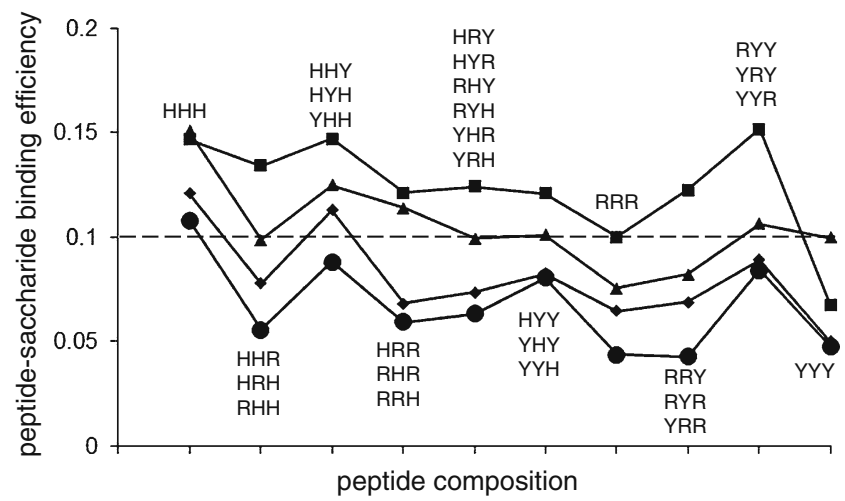

Figure 3. Binding efficiency of tripeptides for each disaccharide as a function of peptide amino acid composition. The traces connecting the square, triangle, diamond, and circle data points correspond to data for melibiose, leucrose, trehalose, and palatinose, respectively

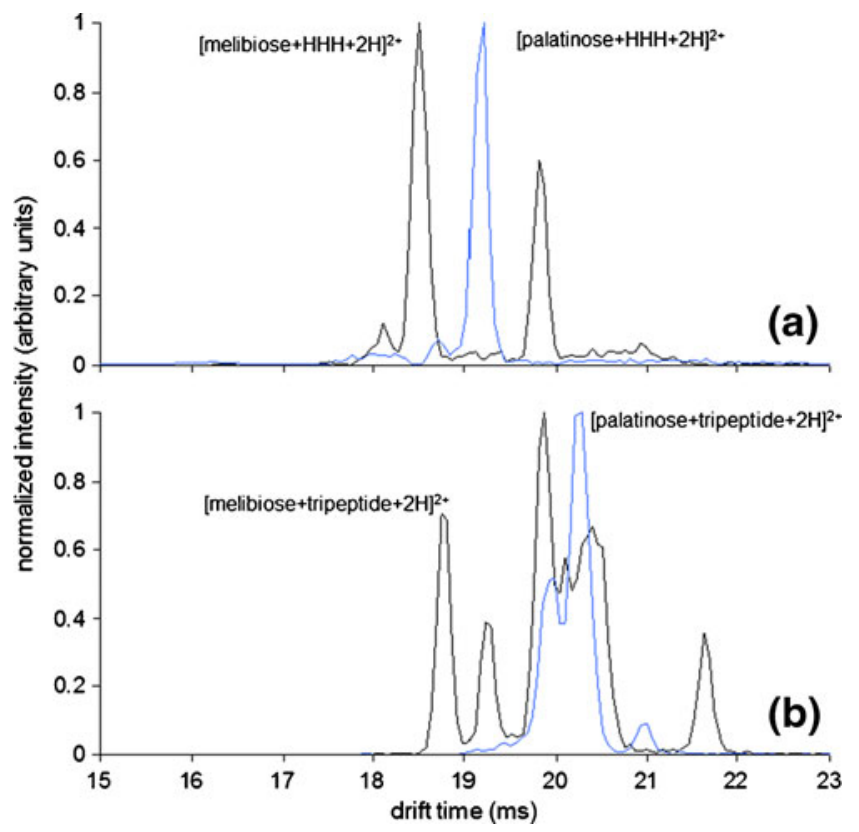

Figure 4. Drift time distributions for (a) doubly protonated complexes of trihistidine with palatinose and melibiose and (b) doubly protonated complexes of six possible tripeptides (containing one histidine, tyrosine, and arginine residue each) with palatinose and melibiose

\section{Resolution of Disaccharide Isomers that are not Resolved as $[\mathrm{M}+\mathrm{Na}]^{+}$Ions}

Recalling that sodiated palatinose and melibiose ions were not resolved from one another, these species present a good test case for assessing our shift reagent approach. Integration of the data across the ten unique $\mathrm{m} / \mathrm{z}$ values for the tripeptide-disaccharide complex ions produces drift time distributions for each disaccharide in its complexed form. Of these drift distributions, eight spectra do not show any improvement in resolving the isomers. Two distributions, however, show evidence that peptide reagents can significantly improve the mobility separation (Figure 4). The doubly protonated complex between trihistidine and these disaccharides has a unique $\mathrm{m} / \mathrm{z}$, making its assignment and drift distribution unambiguous. The $[\text { palatinose }+\mathrm{HHH}+2 \mathrm{H}]^{2+}$ ions appear as a single sharp peak in the drift time distribution, whereas the [melibiose $+\mathrm{HHH}+2 \mathrm{H}]^{2+}$ distribution splits into a doublet, roughly centered about, and baseline-resolved from, the other peak. The origin of this doublet is intriguing, given that the sodiated disaccharides and doubly protonated trihistidine ions all predominantly appear as single features upon IMS-MS analysis (data for trihistidine not shown). One might speculate that the two peaks observed for the [melibiose $+\mathrm{HHH}+2 \mathrm{H}]^{2+}$ distribution originates from two different binding modes of trihistidine; one peak corresponds to trihistidine binding to the galactose subunit, and the other to trihistidine associating with the glucose moiety. This interpretation is also consistent with the singlet observed for [palatinose $+\mathrm{HHH}+2 \mathrm{H}]^{2+}$, as palatinose contains only one pyranose unit for binding. Its fructose subunit, as discussed above, presumably does not present a binding domain for the peptide; thus, only a single binding mode exists for palatinose. 


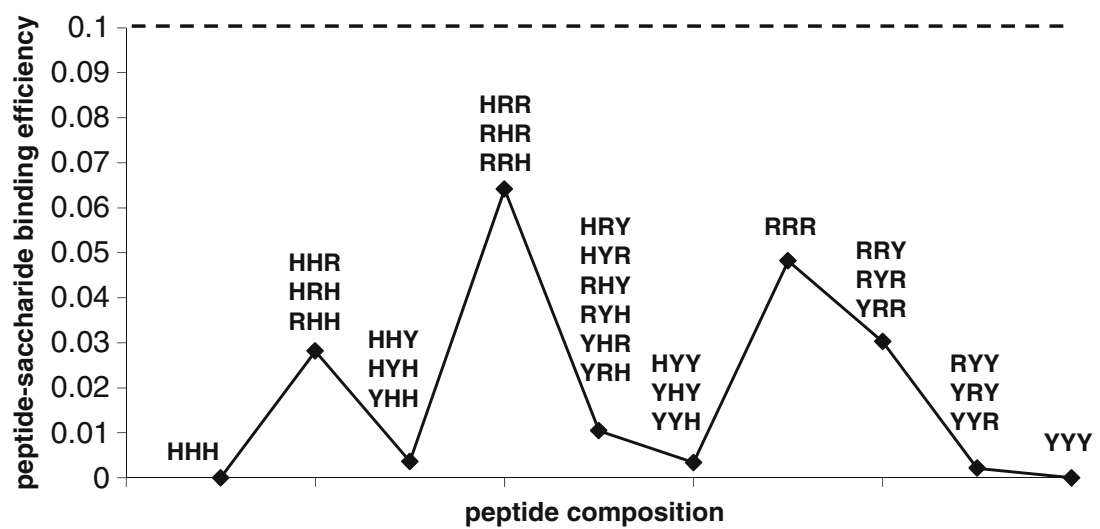

Figure 5. Binding efficiency plot for the tripeptide library and $\mathrm{N}$-acetylmuramic acid (MurNAc)

The other peptide sequence that shows promise as a shift reagent contains one histidine, tyrosine, and arginine residue each (the same stoichiometry found in the recognition protein's binding cleft). Figure $4 \mathrm{~b}$ shows IMS spectra for each disaccharide with these six different peptide isomers. The two distributions are so drastically different that it seems likely one of the six peptide isomers may be useful as a shift reagent. Also, complexes with palatinose give rise to a distribution containing at least six different structures, whereas only three features can be resolved for the complexes with melibiose. That fewer features are observed for palatinosetripeptide complexes than melibiose-tripeptide complexes is also consistent with our hypothesis regarding the possibility of selective binding to pyranose subunits.

It is interesting to consider the extent to which the tripeptide or disaccharide subunits dictate the overall structure of the complex. The interaction between the peptide and carbohydrate subunits to determine favored complex structures, though not well-understood at this time, is likely to be more effective than the metal cation-based strategies to resolve isomeric species in mobility separations. The results for trihistidine-disaccharide complexes demonstrates that isomeric target molecules can interact differently (and perhaps specifically) with an appropriate peptide reagent. Metal cations, on the other hand, tend to collapse saccharide structures (at least in 1:1 complexes of oligosaccharide:metal cation) to conformations with very similar cross sections [7].

\section{Tripeptide Binding Properties for Other Saccharide-Based Analytes}

To consider binding efficiencies for other saccharides, we have selected two model compounds to better characterize this set of peptide reagents. We begin with $N$-acetylmuramic acid (MurNAc) because it is the natural ligand for PGRP, the protein this library is based upon. Thus, one would expect the library, or some subset of sequences therein, to demonstrate high binding efficiency for this molecule. Additionally, we have examined binding of the library to maltohexaose to examine the potential stoichiometries of complexes formed between the tripeptide library and saccharide molecules. Maltohexaose contains six $\alpha(1 \rightarrow 4)$ linked glucose subunits, and thus provides a larger saccharide chain compared to the glucose-containing disaccharides and may be able to accommodate multiple peptide adducts.

Complexes formed between MurNAc and peptide reagents demonstrate similar stoichiometries compared to the disaccharide system. [MurNAc + tripeptide $+2 \mathrm{H}]^{2+}$ ions were found to be the favored binding states for tripeptide-MurNAc complexes. Doubly protonated complexes with two and three MurNAc ligands, as well as $[\text { MurNAc }+ \text { tripeptide }+\mathrm{H}]^{+}$ions, were also detected. The tripeptide-MurNAc complexes differ from the disaccharide system in two significant ways and are summarized in Figure 5. First, the binding efficiency of the peptides for the MurNAc was much lower than anticipated; none of the peptides even approach the $10 \%$ level that estimates the maximum binding efficiency for nonspecific interactions. One possible factor for the relatively low binding efficiency of MurNAc is given by the intense $[\text { MurNAc }+\mathrm{H}]^{+}$peak in the mass spectrum (data not shown). This indicates that, despite containing a carboxylic acid and acetylated nitrogen moieties, the molecule surprisingly protonates quite readily. Therefore, unlike the neutral disaccharide ligands, interactions between MurNAc and the tripeptides will include significant electrostatic repulsion,

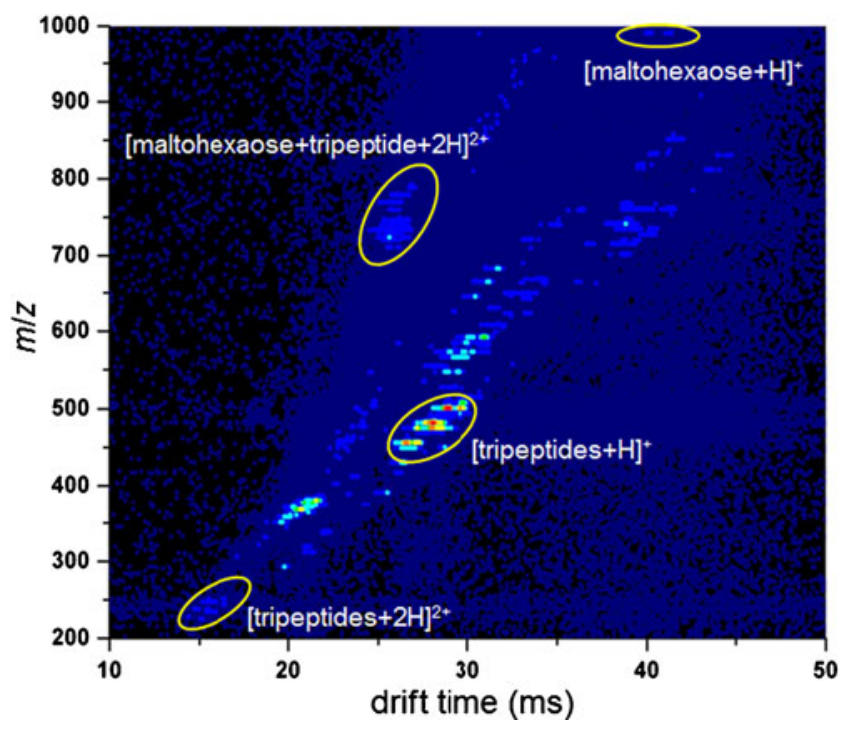

Figure 6. IMS-MS nested 2D plot generated upon analysis of maltohexaose with the tripeptide library 
leading to increased destabilization upon entering the gas phase. It is also possible that protonation presents a competing pathway for ionization, thus lowering the abundance of tripeptide-MurNAc complexes observed. The tripeptide-disaccharide system also demonstrates decreased apparent binding efficiency when competing ionization pathways are available, as when sprayed from solutions containing $2 \mathrm{mM}$ sodium acetate (data not shown). Secondly, the tripeptide-MurNAc complexes differ from those with disaccharides in the peptide composition that binds most favorably. Whereas the disaccharides generally bound to histidine-rich sequences favorably, MurNAc appears to complex more readily with arginine-rich sequences. The variability of preferred binding compositions for different saccharide-based ligands suggests that further generations of this library can be tuned to favor particular analyte substrates.

Results for the analysis of maltohexaose with the peptide library (Figure 6) indicate that the $[\mathrm{M}+\text { tripeptide }+2 \mathrm{H}]^{2+}$ species continues to be the preferred binding mode. It is interesting that, unlike the disaccharide analysis, this system does not generate a triply protonated series of ions. Presumably, the third proton in those complexes was stabilized by the clustering of additional disaccharide units; $[\mathrm{M}+\text { tripeptide }+3 \mathrm{H}]^{3+}$ ions were not observed for 1:1 tripeptide:disaccharide complexes. The maltohexaose system does not demonstrate such clustering and, thus, the lack of a triply protonated series of ions is consistent with the requirement of cluster formation to accommodate a third proton. Also absent from these data is the presence of any complexes with a binding stoichiometry greater than 1 (tripeptide:saccharide). This suggests that saccharidebased analytes below $1000 \mathrm{Da}$ in mass do not bind multiple tripeptide reagents.

\section{Conclusions}

A 27-component combinatorial library of peptides was designed based on the nonadjacent histidine, tyrosine, and arginine residues associated with the glycan recognition properties of a protein. These short linear sequences retain sufficient binding properties as to be analytically useful in the IMS-MS separations of carbohydrates. Compared with estimated binding efficiencies for nonspecific protein-saccharide interactions, [39] the peptide reagents here appear to interact with disaccharides with some specificity. The preferential binding that is observed among a set of isomeric species allows these complexes to be resolved by IMS-MS analysis.

The tripeptide charge-carriers have inherent structure that is likely important in dictating the overall structure of complexes with different isomeric forms of an analyte. This is supported by the evidence suggesting that tripeptide-disaccharide binding is influenced by characteristics of both the peptide (regarding its composition) and the disaccharide (regarding its linkage and subunit ring type). We note that the utility of these reagents appears to be susceptible to competing ionization pathways and may be limited by peptide:analyte stoichiometry (at least for analytes below molecular weight of $1000 \mathrm{Da}$ ). The cases reported on here are preliminary investigations into a limited number of biologicallyinspired peptide-carbohydrate interactions to be utilized in analytical techniques; clearly, more examples are needed to produce a readily applicable methodology. Nonetheless, the results presented here have shown that simulation of specific protein-ligand interactions using short peptide reagents is an analytically useful approach for resolving isomeric molecules.

\section{Acknowledgments}

The authors acknowledge support for this work by grants from the Indiana University METACyt initiative funded by the Lilly Endowment and from the National Institutes of Health (1RC1GM090797-01).

\section{References}

1. Hagglund, P., Bunkenborg, J., Elortza, F., Jensen, O.N., Roepstorff, P.: A new strategy for identification of $\mathrm{N}$-glycosylated proteins and unambiguous assignment of their glycosylation sites using HILIC enrichment and partial deglycosylation. J. Proteome Res. 3, 556-566 (2004)

2. Zielinska, D.F., Gnad, F., Wisniewski, J.R., Mann, M.: Precision mapping of an in vivo N-Glycoproteome reveals rigid topological and sequence restraints. Cell 141, 897-907 (2010)

3. Viner, R.I., Zhang, T., Second, T., Zabrouskov, V.: Quantification of post-translationally modified peptides of bovine alpha-crystallin using tandem mass tags and electron transfer dissociation. J. Proteom. 72, 874-885 (2009)

4. An, H.J., Lebrilla, C.E.: Structure elucidation of native N- and OLinked glycans by tandem mass spectrometry (Tutorial). Mass Spectrom. Rev. (2010). doi:10.1002/mas.20283

5. Ashline, D.J., Lapadula, A.J., Liu, Y.-H., Lin, M., Grace, M., Pramanik, B., Reinhold, V.N.: Carbohydrate structural isomers analyzed by sequential mass spectrometry. Anal. Chem. 79, 3830-3842 (2007)

6. Bohrer, B.C., Merenbloom, S.I., Koeniger, S.L., Hilderbrand, A.E., Clemmer, D.E.: Biomolecule analysis by ion mobility spectrometry. Annu. Rev. Anal. Chem. 1, 10.1-10.35 (2008)

7. Lee, S., Wyttenbach, T., Bowers, M.T.: Gas phase structures of sodiated oligosaccharides by ion mobility ion chromatography methods. Int. J. Mass Spectrom. Ion Process. 167/168, 605-614 (1997)

8. Liu, Y., Clemmer, D.E.: Characterizing oligosaccharides using injectedion mobility/mass spectrometry. Anal. Chem. 69, 2504-2509 (1997)

9. Lee, D.-S., Wu, C., Hill, H.H.: Detection of carbohydrates by electrospray ionization ion mobility spectrometry following microbore highperformance liquid chromatography. J. Chromatogr. A 822, 1-9 (1998)

10. Clowers, B.H., Dwivedi, P., Steiner, W.E., Bendiak, B., Hill, H.H.: Separation of sodiated isobaric disaccharides and trisaccharides using electrospray ionization-atmospheric pressure ion mobility-time of flight mass spectrometry. J. Am. Soc. Mass Spectrom. 16, 660-669 (2005)

11. Jin, L., Barran, P.E., Deakin, J.A., Lyon, M., Uhrin, D.: Conformation of glycoaminoglycans by ion mobility mass spectrometry. Phys. Chem. Chem. Phys. 7, 3464-3471 (2005)

12. Dwivedi, P., Bendiak, B., Clowers, B.H., Hill, H.H.: Rapid resolution of carbohydrate isomers by electrospray ionization ambient pressure ion mobility spectrometry-time-of-flight mass spectrometry (ESI-APIMSTOFMS). J. Am. Soc. Mass Spectrom. 18, 1163-1175 (2007)

13. Plasencia, M.D., Isailovic, D., Merenbloom, S.I., Mechref, Y., Clemmer, D.E.: Resolving and assigning N-Linked glycan structural isomers from ovalbumin by IMS-MS. J. Am. Soc. Mass Spectrom. 19, 1706-1715 (2008)

14. Yamagaki, T., Sato, A.: Isomeric oligosaccharides analysis using negative-ion electrospray ionization ion mobility spectrometry combined with collision-induced dissociation MS/MS. Anal. Sci. 25, 985988 (2009)

15. Williams, J.P., Grabenauer, M., Holland, R.J., Carpenter, C.J., Wormald, M.R., Giles, K., Harvey, D.J., Bateman, R.H., Scrivens, J. H., Bowers, M.T.: Characterization of simple isomeric oligosaccharides and rapid separation of glycan mixtures by ion mobility mass spectrometry. Int. J. Mass Spectrom. 298, 119-127 (2010)

16. Fenn, L.S., McLean, J.A.: Structural resolution of carbohydrate positional and structural isomers based on gas-phase ion mobility mass spectrometry. Phys. Chem. Chem. Phys. 13, 2196-2205 (2011) 
17. Asbury, G.R., Hill, H.H.: Using different drift gases to change separation factors $(\alpha)$ in ion mobility spectrometry. Anal. Chem. 72, 580-584 (2000)

18. Matz, L.M., Hill, H.H., Beegle, L.W., Kanik, I.: Investigation of drift gas selectivity in high resolution ion mobility spectrometry with mass spectrometry detection. J. Am. Soc. Mass Spectrom. 13, 300-307 (2002)

19. Fernandez-Maestre, R., Wu, C., Hill, H.H.: Using a buffer gas modifier to change separation selectivity in ion mobility spectrometry. Int. J. Mass Spectrom. 298, 2-9 (2010)

20. Fenn, J.B., Mann, M., Meng, C.K., Wong, S.F., Whitehouse, C.M.: Electrospray ionization for mass spectrometry of large biomolecules. Science 246, 64-71 (1989)

21. Zhang, Y., Go, E.P., Jiang, H., Desaire, H.: A novel mass spectrometric method to distinguish isobaric monosaccharides that are phosphorylated or sulfated using ion-pairing reagents. J. Am. Soc. Mass Spectrom. 16, 1827-1839 (2005)

22. Zhang, Y., Jiang, H., Go, E.P., Desaire, H.: Distinguishing phosphorylation and sulfation in carbohydrates and glycoproteins using ion-pairing and mass spectrometry. J. Am. Soc. Mass Spectrom. 17, 1282-1288 (2006)

23. Cocinero, E.J., Carcabal, P., Vaden, T.D., Simons, J.P., Davis, B.G.: Sensing the anomeric effect in a solvent-free environment. Nature $\mathbf{4 6 9}$, 76-80 (2011)

24. Von Seggern, C.E., Cotter, R.J.: Study of peptide-sugar non-covalent complexes by infrared atmospheric pressure matrix-assisted laser desorption/ionization. J. Mass Spectrom. 39, 736-742 (2004)

25. Nimrichter, L., Burdick, M.M., Aoki, K., Laroy, W., Fierro, M.A., Hudson, S.A., Von Seggern, C.E., Cotter, R.J., Bochner, B.S., Tiemeyer, M., Konstantopoulos, K., Schnaar, R.L.: E-selectin receptors on human leukocytes. Blood 112, 3744-3752 (2008)

26. Guan, R., Roychowdury, A., Ember, B., Kumar, S., Boons, G.-J., Mariuzza, R.A.: Crystal structure of a peptidoglycan recognition protein (PGRP) complex with a muramyl tripeptide from gram-positivie bacteria. J. Endotoxin Res. 11, 41-46 (2005)

27. Vollmer, W., Blanot, D., de Pedro, M.A.: Peptidoglycan structure and architecture. FEMS Microbiol. Rev. 32, 149-167 (2008)
28. Hilderbrand, A.E., Myung, S., Clemmer, D.E.: Exploring crown ethers as shift reagents for ion mobility spectrometry. Anal. Chem. 78, 6792$6800(2006)$

29. Howdle, M.D., Eckers, C., Laures, A.M.F., Creaser, C.S.: The use of shift reagents in ion mobility-mass spectrometry: Studies on the complexation of an active pharmaceutical ingredient with polyethylene glycol excipients. J. Am. Soc. Mass Spectrom. 20, 1-9 (2009)

30. Lebl, M., Krchnak, V.: Synthetic peptide libraries. Methods Enzymol. 289, 336-392 (1997)

31. Wellings, D.A., Atherton, E.: Standard Fmoc protocols. Methods Enzymol. 289, 44-67 (1997)

32. Koeniger, S.L., Merenbloom, S.I., Valentine, S.J., Jarrold, M.F., Udseth, H.R., Smith, R.D., Clemmer, D.E.: An IMS-IMS analogue of MS-MS. Anal. Chem. 78, 4161-4174 (2006)

33. Shaffer, S.A., Tang, K.Q., Anderson, G.A., Prior, D.C., Udseth, H.R., Smith, R.D.: A novel ion funnel for focusing ions at elevated pressure using electrospray ionization mass spectrometry. Rapid Commun. Mass Spectrom. 11, 1813-1817 (1997)

34. Mason, E.A., McDaniel, E.W.: Transport Properties of Ions in Gases. Wiley, New York (1988)

35. Meslah, M.F., Hunter, J.M., Shvartsburg, A.A., Schatz, G.C., Jarrold, M.F.: Structural information from ion mobility measurements: Effects of the long-range potential. J. Phys. Chem. 100, 16082-16086 (1996)

36. Wyttenbach, T., von Helden, G., Batka, J.J., Carlat, D., Bowers, M.T.: Effect of the long-range potential on Ion mobility measurements. J. Am. Soc. Mass Spectrom. 8, 275-282 (1997)

37. Hoaglund, C.S., Valentine, S.J., Sporleder, C.S., Reilly, J.P., Clemmer, D.E.: Three-dimensional ion mobility/TOFMS analysis of electrosprayed biomolecules. Anal. Chem. 70, 2236-2242 (1998)

38. Sapir, L., Harries, D.: Linking trehalose self-association with binary aqueous solution equation of state. J. Phys. Chem. B 115, 624-634 (2011)

39. Wang, W., Kitova, E.N., Klassen, J.S.: Non-specific protein-carbohydrate complexes produced by nanoelectrospray ionization: Factors influencing their formation and stability. Anal. Chem. 77, 3060-3071 (2005)

40. Van der Heijeden, A.M., Lee, T.C., van Rantwijk, F., van Bekkum, H.: Glycosidation of fructose-containing disaccharides using MCM-41 material as the catalyst. Carbohydr. Res. 337, 1993-1998 (2002) 